\title{
Malicious Attack Identification Using Deep Non Linear Bag-of-Words (FAI-DLB)
}

\author{
Dr.E.Arul ${ }^{\mathrm{a}, 1}$, A.Punidha ${ }^{\mathrm{b}}$, K.Gunasekaran ${ }^{\mathrm{c}}$, P Radhakrishnan ${ }^{\mathrm{c}}$, VD Ashok Kumar ${ }^{\mathrm{d}}$ \\ $a, 1$ \\ Assistant Professor, Department of IT, Coimbatore Institute of technology, \\ Coimbatore, Tamilnadu, India \\ Assistant Professor, Department of CSE, Coimbatore Institute of technology, \\ Coimbatore, Tamilnadu, India \\ Assistant Professor, Department of CSE, Panimalar Enginnering College, Chennai, \\ Tamulnadu, India \\ ${ }^{d}$ Research Scholar, Department of CSE, St.Peter's University, Chennai, \\ Tamilnadu, India
}

\begin{abstract}
Online media have flourished in modern years to connect with the world. Most of those stuff users share on blogs like facebook, twitter and many other are pessimistic or just middle spirited. Further, an increasingly professional anti spyware technologies are dependent on Machine Learning(ML) technology to secure malicious consumers. Over the past few years, revolutionary learning approaches have yielded remarkable outcomes and have immediately generated photos, characters and text interpretations of dynamic weak points. The Purple consumer frequency makes the troll and attacker aim an enticing one. The users will learn the controversial topics and techniques used by malware from articles with ties to harmful material and bogus applications. It is essential to build and customize a lot of potential functionality in vulnerability and application developers around the world. To represent a public web firmware assault with deep logistic inference using Extreme Spontaneous Tree (FAI-DLB). A corresponding output device is named harmful or benign by training an FAI-DLB with different modulation clustered with such a normal or anomalous API. It was therefore equipped to locate a suspicious sequence in unidentified firmware of FAI Deep LB. The outcome demonstrates a good actual meaning of $96.25 \%$ and a low spyware assault of $0.03 \%$.
\end{abstract}

Keywords. IoT, Backdoors, Malware, API calls, Deep Learning, Random Forest, Firmware

\section{Introduction}

The Malicious software is classified into many groups, depending on the manner the program is implemented as well as the direction it travels[12]. A virus or computer virus that is self replicated by exporting itself to another application. A Malware triggering 
may be a harmful mail connection or an inexperienced person loading a Flash memory stick into a device. The machine is corrupted by the duplication of the malware. A Worm, malware that travels around the system by reproducing itself by user interference. Trojan a sort of ransom ware that resides in a java script code and runs on a device next to it. A Security hole malware that provides secret, permanent exposure to an undiscovered device, which unlocks the door to a machine with little manual intervention. Infectors of the file:Create mutual infections arrive to recognized file formats, such as.com or.exe. These are implemented when the programs are launched.

\section{LITERATURESURVEY}

P. Dewan ,The increase in user engagement on online social networks ( OSNs) is seen as a consequence of a news case[1]. The computer hackers are using this drive to expand illegal code to destroy the reputation of the scheme, cause revenue damage and diminish user interface. The whole article established a number of community comments on instagram, produced over 4.4 million, and 11217 malevolent posts that included URLs. In eight headlines-making events (disaster warning, terrorist attacks etc.)[7], almost all of the offensive software that is actually trying to evade Facebook strategies has been discovered by third parties and web apps and about half of any and all quality content has been identified. The REST API and a browser-based plug-in are developed to detect suspicious posts on social media in near real - time.

R. J. Hada ,In recent years, social media growth has increased considerably when too many users visit social media across mobile media with the substantial emergence of knowledgeable smart phones and rapidly increasingly digital modern networks. A massive social networking site (OSN) on a database is always costly or even difficult to implement. Lateral scale up, where OSN is subdivided and distributed on a range of cheap servers, is an economical strategy. They are researching the issue of controlled segmentation of OSNs at minimal cost throughout this work. This blends the clusters accordingly and transfers clusters of the very same scale, thus raising the overall expense based on cross server traffic. We introduce and test all architectures on Twitter, Arxiv, tackle complex, Aim's solving wide range OSN repositories [4].

\section{Delineation of Supervised Deep Learning Vector Quantization to Detect IoT Malware}

A new of the tranquil runtime environments in NLP is its mock-up framework. It provides an illustrative template of both the document by monitoring every phrase's frequency of occurrence. It can be subsequently used as message clustering algorithm characteristics. For this template, you just consider particular terms and apply a particular moral relativism score for each term. In a vocabulary of feelings[12], this moral relativism value can be tested. If the overall score is negative, the text will be categorized as pessimistic and the message as beneficial. The written language and pronunciation are not taken into consideration. This is easy to make, but also less precise. This phrase provides the identical performance with unigrams, but never through the bigrams model + unigrams. 

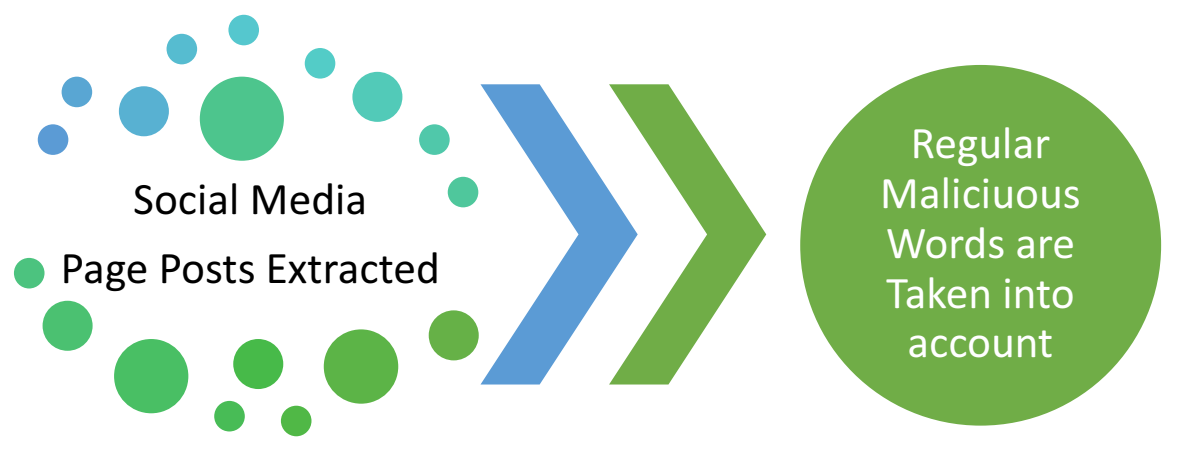

Preprocessing Normal HTML

Tag remove and take the words or sentences contains the linkable Fields

Identified Threadable Strings

in the linkable

Figure 1:Delineation of the Fuzzy zip lock-of-words to identify fake links in social post

Indistinct logic is as followed to develop a classifier zip lock-of-words in various post present in the social media page.

- $\quad$ Fake_List $=[]$,For each review in the training set:

- Only at give up of each evaluation, deprive this same non - ascii character "vector" ,Place a space before and after each of the following characters: .,()[]:;" (This prevents sentences like "I like this book.It is engaging" being interpreted as [“Click", "Post", "Share", "Install It", "Lucky", "love you”].)

- Divide into gaps, categorize the file. Erase trinkets that are only a platform, vacant pair, or dot label.

- For Fake_List, add the keys. Already Fake_List includes the entire set of parameters.

- In JavaScript response item, spot list Fake_List. This monitor currently includes both terms and their coefficients. The utmost collective function can also be used to sort your posts.

This pattern concentrates solely on terms, or often on a sequence of words, but typically fails to answer the so called "framework." The method term bag generally includes a wide list which is typically best understood as a kind of "directional," which have been regarded as experiencing words. Each of these phrases has its own "importance" in document. Usually the factors are all incorporated, resulting in a perception evaluation. There are several different equations to attach and extract, however this template concentrates mostly on terms and doesn't try to grasp the function of knowledge actually. 


\section{EXPERIMENTAL RESULTS AND COMPARISON}

This template tries to make the computer recognize the frameworks, meaning of the statements and concentrates a little more on progression of a series. Typically, the computer needs to grasp the grammatical structures in this framework[24]. The natural language processing (NLP) technology for this purpose is used to tag parts of the language, named entities or more. It is not to search only for target words to truly understand the 'language' of the text. With using a tagger or an encoding template, it is essential to clarify the corpus for stand. The further terms, more and more papers are represented, and so it is necessary to minimize the words to those that are assumed to be significant predictors. This is not easy to learn and sometimes various theories about developing an using of must be checked. It can build in table 1 a repertoire as a reference, a database that makes it easy to check terms and their counters. The studies illustrate that we already have a 55,125 choosing the right words. Within the opinion pieces, we can even see a list of the best 50 words. Remember that even these comments in the test set were focused on another terminology.

During the analysis it is found that the frequency of matching word in different malicious posts were given as follows:

[('<a>', 1213), ('share', 2146), ('click', 4826), ('like', 3201), ('sh', 2262), ('exe', 2080), ('time', 4321), ('<iframe>', 1107), ('love click', 1873), ('offer', 1844), (best', 1824), ('tick', 5452), ('like', 1735), ('get', 1214), ('character', 1233), ('my show', 5453), ('level', 5523), ('see', 4212), ('way', 3243), ('cricket', 5642), ('score', 1231), ('really', 4534), ('book', 2133), ('threat', 1233), ('plot', 1288), ('people', 3219), ('could', 1248), ('new', 1248), ('scene', 1241), ('download', 1238), ('never', 1201), ('best', 4323), ('update', 4321), ('songs', 1135), ('man', 4241), ('many', 1321), ('doesnt', 4323), ('know', 1092), ('dont', 1421), ('hes', 1024), ('great', 1014), ('another', 992), ('action', 985), ('love', 977), ('us', 3212), ('go', 952), ('enter', 3213), ('age', 321), ('group', 678), ('pills', 567)].

The algorithm resolves documentation in the terminology and delete all small incidence terms along with once maybe twice in all comments. For instance, just the signatures which occur 2 or even more times in every analysis can be outlined in the following excerpt. By using the instance described with for this inclusion, the language is reduced from 55,125 to 32,121 words by either a little over twice its duration. This represents a much more expressionistic document than conventional techniques such as bag-of words, where connections among both phrases are overlooked or compelled into bigrams or trigrams.

Table 1. Suggested Interpretations Deep Zip-Lock against known malicious strategies

\begin{tabular}{lcccc}
\hline Methods & $\begin{array}{l}\text { Amount of } \\
\text { Uncoveringof } \\
\text { hazard files }\end{array}$ & $\begin{array}{c}\text { Exact Quantity } \\
(\%)\end{array}$ & $\begin{array}{l}\text { Imprecise } \\
\text { Professed }\end{array}$ & $\begin{array}{l}\text { Imprecise } \\
\text { SupposedPart (\%) }\end{array}$ \\
\hline P. Dewan & 923 & 83.75 & 179 & 0.13 \\
$\begin{array}{l}\text { R. J. Hada } \\
\text { Proposed SM- } \\
\text { DLB }\end{array}$ & 991 & 89.92 & 111 & 0.08 \\
\hline
\end{tabular}




\section{CONCLUSION AND FUTURE WORK}

In social networking sites like: Facebook, Google +, and other users in Twitter, manipulative attack can be used. Many of these participants really are not customers, partners or experienced men. The goal is to harm the entity or the organization clearly. The bulk of injected vulnerabilities into another computer system utilize some popular social networking, Platform-related calls to execute recognized suspicious attacks. The Trojan grabs your personal data and sends it over to the cyber database, distributes suspicious phishing, and uses the full maximum throughput of any framework. The cropping up used explicitly for malevolent as well as grouped API security software calls performing spamware procedure in this future framework. A FAI-DLB training set has been used finally to verify whether some harmful commands have more similarities. The consequence is a real positive result of $97.36 \%$ of the different social network software assaults and a fake certainly develop rating of $0.02 \%$. In long term, the whole process will be completed with different APIs that enables malevolent systems integration.

\section{References}

[1] P. Dewan and P. Kumaraguru, "Towards automatic real time identification of malicious posts on Facebook," 2015 13th Annual Conference on Privacy, Security and Trust (PST), Izmir, 2015, pp. 85-92, doi: 10.1109/PST.2015.7232958.

[2] J. Zhang, B. Dong and P. S. Yu, "Deep Diffusive Neural Network based Fake News Detection from Heterogeneous Social Networks," 2019 IEEE International Conference on Big Data (Big Data), Los Angeles, CA, USA, 2019, pp. 1259-1266, doi: 10.1109/BigData47090.2019.9005556.

[3] R. J. Hada, H. Wu and M. Jin, "Scalable Minimum-Cost Balanced Partitioning of Large-Scale Social Networks: Online and Offline Solutions," in IEEE Transactions on Parallel and Distributed Systems, vol. 29, no. 7, pp. 1636-1649, 1 July 2018, doi: 10.1109/TPDS.2017.2694835.

[4] Kakisim, Arzu, Nar, Mert\&Sogukpinar, Ibrahim “. Metamorphic malware identification using enginespecific patterns based on co-opcode graph" Computer Standards \& Interfaces. 2020. doi:71. 103443. 10.1016/j.csi.2020.103443.

[5] Miserendino, Scott, Peters, Ryan, Klein, Robert, Kaloroumakis, Peter. " System and method for insitu classifier retraining for malware identification and model heterogeneity.", US20200401941, 2018

[6] Irofti, Paul, Băltoiu, Andra. "Malware Identification with Dictionary Learning", 2019.. doi:10.23919/EUSIPCO.2019.8903043.

[7] Chen, Rong, Li, Yangyang, Fang, Weiwei. "Android Malware Identification Based on Traffic Analysis", 2019. doi:10.1007/978-3-030-24274-9_26.

[8] Luo, Xiong, Li, Jianyuan, Wang, Weiping, Gao, Yang, Zhao, Wenbing. "A Malware Identification and Detection Method Using Mixture Correntropy-Based Deep Neural Network”, 2019. doi:10.1007/978981-15-1922-2 23.

[9] Miserendino, Scott \& Peters, Ryan \& Klein, Robert \&Kaloroumakis, Peter. (2018). System and method for in-situ classifier retraining for malware identification and model heterogeneity.

[10] Lopes, Joao, Serrao, Carlos, Nunes, Luis, Almeida, Ana, Oliveira, Joao. "Overview of machine learning methods for Android malware identification”, pp 1-6., 2019.doi:s 10.1109/ISDFS.2019.8757523.

[11] Hussain, Syed, Ahmed, Usman, Liaquat, Humer, Mir, Shib, Zaman, Noor , Humayun, Mamoona, "Intelligent Malware Identification for Android Platform" pp: 1-6., 2019 doi:10.1109/ICCISci.2019.8716471.

[12] Sharma, Anshul\& Singh, Sanjay. " A novel approach for early malware detection. Transactions on Emerging Telecommunications Technologies”.2021. doi.10.1002/ett.3968. 